\title{
Prevention of gravidic endothelial hypertension by aspirin treatment administered from the 8th week of gestation
}

\begin{abstract}
Abdelouahab Bakhti ${ }^{1}$ and Daniel Vaiman ${ }^{2}$
The aim of this study was to evaluate whether low doses of aspirin ( $100 \mathrm{mg}$ per day) administered to a homogeneous population of women early (8-10 weeks) during their first pregnancy improved the outcome of gestation hypertensive disorders. This study was performed at the Blida Hospital, where many early deliveries and pregnancy complications are observed. A total of 164 women were either treated (82) or used as controls (82). Treatment increased the gestation length by 12 days on average, thus triggering an approximate 150-g increase in newborn weight. This consistently improved the outcome for all patients with respect to all parameters investigated. Overall, the relative risk of developing hypertensive disorders of gestation was reduced to 0.07 (confidence interval=0.01-0.51). In our series, we did not observe deleterious consequences for the fetus (teratogenicity and fetotoxicity) or adverse outcomes for the mothers. Despite the limited number of patients analyzed, the present study is one of the largest investigating early aspirin treatment of gestational hypertensive diseases. In addition, the time of aspirin administration is among the earliest yet examined. The data tend to confirm the results obtained from other cohorts on the overall benefit of aspirin treatment for gestational disorders. In the future, molecular or ultrasonographic markers of these diseases could help to screen patients before applying the treatment.
\end{abstract}

Hypertension Research (2011) 34, 1116-1120; doi:10.1038/hr.2011.111; published online 1 September 2011

Keywords: early aspirin prescription; first gestation; hypertensive disorders of pregnancy; IUGR; pre-eclampsia

\section{INTRODUCTION}

Pre-eclampsia and other hypertensive diseases of pregnancy are associated with systemic endothelial dysfunction causing materno-fetal morbidity and mortality. These diseases occur de novo (that is, in patients who did not present hypertensive symptoms before gestation), and they can only worsen with the course of pregnancy. The hypertension in pre-eclampsia is associated with proteinuria. In the most severe cases, pre-eclampsia necessitates pre-term extraction of the fetus and is therefore a major cause of prematurity. ${ }^{1,2}$ It is estimated that overall, $16 \%$ of mothers develop a hypertensive disease of pregnancy. In preeclampsia, this has been associated with visceral alterations that later affect the mother's well-being; for example, it has recently been shown that mothers who experienced pre-eclampsia have a higher risk of undergoing a renal transplant later in life, with a relative risk of $4.7 .^{3}$

These hypertensive diseases are of placental origin, because the delivery is generally followed by the disappearance of symptoms, often in less than 1 week. ${ }^{4}$ The link between placental dysfunction and the mother's syndrome has been quite elusive until the last 10 years or so, when the hypothesis of residual syncytiotrophoblastic material from placental shedding ${ }^{5}$ or placenta microparticles ${ }^{6}$ was put forward. This material can release vasoactive substances to the maternal blood flow, which in turn could durably affect the maternal endothelium throughout the body. Indeed, specific alterations of protein content in the mother's serum in pre-eclampsia have now been documented in several proteomic studies. ${ }^{7,8}$ Pro-inflammatory molecules such as C-reactive protein were consistently increased in the maternal serum along with coagulation factors such as gamma fibrinogen, various SERPINs of the A and C clades (some possibly synthesized by the placenta $)^{9}$ and lipid transport molecules such as apolipoproteins.

At lower levels than those accessible to proteomic analyses, the discovery of an abnormally increased concentration of soluble receptors for angiogenic factors (sENG, a receptor for ligands of the bone morphogenetic proteins (BMP) family and sFLT1, the soluble receptor for vascular endothelial growth factor (VEGF)) in preeclampsia has likely been the major discovery of recent years with regard to elucidating this disease. ${ }^{10-12}$ These soluble molecules may be generated by changes in the splicing of mRNA, such as the e15 isoform of sFLT1 $1{ }^{13-15}$ and are thought to have the role of 'molecular traps' for their active ligands.

The use of aspirin to ameliorate the symptoms of pregnancy hypertension has been widely documented and debated. Today,

${ }^{1}$ Clinique Hassiba Ben Bouali, Centre Hospitalo-Universitaire de Blida, Blida, Algérie and ${ }^{2}$ Institut Cochin, Institut National de la Santé et de la Recherche Médicale (INSERM) U1016, CNRS UMR8104, and Université Paris Descartes, Paris, France

Correspondence: Dr D Vaiman, Institut Cochin, Genetics and Development Department, 24 rue du Fg St Jacques, Paris 75013, France.

E-mail: daniel.vaiman@inserm.fr

Received 30 November 2010; revised 22 March 2011; accepted 13 April 2011; published online 1 September 2011 
more than 500 Pubmed references appear when 'pre-eclampsia' and 'aspirin' are used as keywords. ${ }^{16}$ As an illustration of this debate, a recent study failed to find any association in a cohort of women followed after in vitro fertilization/intra-cytoplasmic sperm injection (IVF/ICSI). ${ }^{17}$ Recently, however, a meta-analysis that collected data from 11748 women was able to show that the administration of aspirin at low doses before the 16th week of gestation resulted in a relative risk of pre-eclampsia of $0.44 .{ }^{18}$ In contrast, the improvement is completely absent when the treatment is given after the 16th week. This discrepancy is probably the major reason for the existence of contradictory results in many studies of aspirin administration during gestation.

In the present study, we performed phenotypic analysis of patients and newborns seen at the Blida Hospital (Algeria). Women were divided into two groups of 82 aspirin-treated women and 82 nontreated women. In the absence of treatment, numerous materno-fetal complications occur. Pre-term deliveries are more common in Algeria than in Western society. There is therefore a need for drugs such as aspirin that are inexpensive and easily applied. This work is novel compared with previous studies about early treatment with low doses of aspirin in the following two ways: (i) according to the recent metaanalysis by Bujold and et al..$^{18}$ nine studies from 1985 to 2005 satisfied the criteria for eligibility, and all analyzed fewer than 73 treated patients and 63 controls. In this study, we analyzed 82 treated and 82 control patients. (ii) The systematic administration of aspirin at low doses ( $100 \mathrm{mg}$ per day) was continued from the 8 th to the 36 th week of pregnancy, whereas most studies started treatment during weeks 12-14. We observed an average increase of 12 days in the duration of gestation, with beneficial effects on fetus health and weight and a significant decrease in maternal complications induced by hypertensive gestational disease. Our study therefore confirms the idea that aspirin would be a potent help in decreasing the deleterious effects of pre-eclampsia and other hypertensive gestational diseases, once a reliable early marker of at-risk pregnancies is developed.

\section{METHODS}

\section{Patients, ethics and medical treatment}

Two cohorts of 82 patients and 82 controls were analyzed in this study after randomization for treatment (using anonymous sealed envelopes). The control and treated groups were not statistically different with respect to age, body mass index, gestity and parity (Table 1). The patient and the study promoter were not blinded to the conditions of treatment. Inclusion criteria were primigest women consulting before the 10th week of amenorrhea without previous vasculo-renal pathology. Fewer than $5 \%$ of the women were multigest nulliparous (no previous pregnancy of more than 20 weeks of amenorrhea). The gestational age was confirmed between 10 and 13 weeks according to the standards of the French College of Echography. The crown-rump length was measured, gestational age was deduced and fetal growth was followed from 10 to 13 weeks on. Exclusion criteria were: patients consulting later than the 10th week, patients with a counter-indication to the use of aspirin, patients with chronic arterial hypertension before pregnancy and patients with chronic nephropathy or with a known auto-immune disorder. We also excluded twin pregnancies and diabetic women. Despite this selection, the placebo group delivered around 35 weeks, but this was not unusual among the Algerian population. Sample size was calculated for detecting differences at a risk of $5 \%$, a detection power of $80 \%$ and a confidence level of $95 \%$. Based on observations from a preliminary study, it was expected that the initial proportion of gestational hypertension (GHT) of $\sim 16 \%$ would decrease to $\sim 5 \%$ or less. These values led to the estimation that 82 patients were necessary in each group, which explains why we used a cohort of this size (164 patients among $\sim 200$ who were invited to participate). Each patient provided his or her informed consent according to the local ethical regulations. Medical treatment consisted of $100 \mathrm{mg}$ per day of aspirin per os, taken in the evening, before the
Table 1 Characteristics of patients and controls

\begin{tabular}{|c|c|c|c|c|c|c|c|}
\hline & \multicolumn{6}{|c|}{ Age (years) } & \\
\hline & $<19$ & $19-23$ & $24-28$ & $29-33$ & $34-38$ & $39-43$ & \\
\hline Control & 7 & 25 & 27 & 10 & 9 & 4 & $\chi^{2}=0.996$ \\
\hline \multirow[t]{3}{*}{ Treated } & 6 & 26 & 28 & 11 & 8 & 3 & \\
\hline & \multicolumn{5}{|c|}{$B M I\left(\mathrm{~kg} \mathrm{~m}^{-2}\right)$} & & \\
\hline & $22-24$ & $24-25$ & $25-26$ & $26-27$ & $27-28$ & & \\
\hline Control & 15 & 23 & 19 & 14 & 11 & & $\chi^{2}=0.976$ \\
\hline \multirow[t]{3}{*}{ Treated } & 16 & 21 & 20 & 12 & 13 & & \\
\hline & \multicolumn{6}{|c|}{ Gestity } & \\
\hline & \multicolumn{3}{|c|}{ First gestation } & \multicolumn{3}{|c|}{$>1$ gestation } & \\
\hline Control & \multicolumn{3}{|c|}{80} & \multicolumn{3}{|c|}{2} & $\chi^{2}=0.379$ \\
\hline Treated & \multicolumn{3}{|c|}{78} & \multicolumn{3}{|c|}{4} & \\
\hline
\end{tabular}

10th week of amenorrhea; the treatment was systematically arrested at the end of the 36th week. The bleeding delay was checked before drug treatment and controlled 15 days after the start of the treatment. In all 82 treated patients, treatment was continued until the end of the experiment.

\section{Medical follow-up}

Success of the treatment was defined as the absence of gravidic hypertension, pre-eclampsia or other signs of systemic endothelial dysfunction and materialized by the absence of materno-fetal complications. Maternal complications included HTAG systolic blood pressure $>140 \mathrm{~mm} \mathrm{Hg}$ and diastolic blood pressure $>90 \mathrm{~mm} \mathrm{Hg}$, pre-eclampsia defined by a systolic blood pressure $>140 \mathrm{~mm} \mathrm{Hg}$ and diastolic blood pressure $>90 \mathrm{~mm} \mathrm{Hg}$ with $>300 \mathrm{mg}$ per day proteinuria, retroplacental hematoma, HELLP (hemolysis, elevated liver enzymes, low platelet syndrome) and post-partum hemorrhage. For the child, the occurrence of IUGR (intra-uterine growth restriction), prematurity and perinatal mortality was recorded.

\section{Statistical analysis}

Statistical analysis was systematically performed using $\chi^{2}$ contingency tests. A $P$-value of $<0.05$ was considered significant. Odds ratios and confidence intervals were calculated according to the web site http://www.hutchon.net/ ConfidRR.htm, developed by Dr David JR Hutchon.

\section{RESULTS}

The risk of gravid hypertensive disorders was reduced by aspirin administered from the 8th week of gestation

Various types of maternal complications (gravidic hypertension, preeclampsia, retroplacental hematoma, HELLP syndrome and postpartum hemorrhage) were followed in treated and non-treated patients. All these complications were reduced or absent in the treated group (see Figure 1). When all these complications were considered, the difference was highly significant (25 cases of complications vs. 4 , $\left.P=1.7 \times 10^{-5}\right)$. The number of hypertensive gravidic diseases was 13 out of 82 in the non-treated group (four cases of pre-eclampsias and nine cases of GHT), as compared with only one case of GHT among the 82 treated patients ( 15.9 vs. $1.2 \%, P<0.0008)$. The relative risk was estimated at 0.07 (95\% confidence interval: 0.01-0.51). Taking each condition separately, the risk of GHT was significantly decreased $(P=0.009)$, as were the risks of pre-eclampsia $(P=0.04)$ and retroplacental hematoma $(P=0.04)$. The risks of HELLP and post-partum 


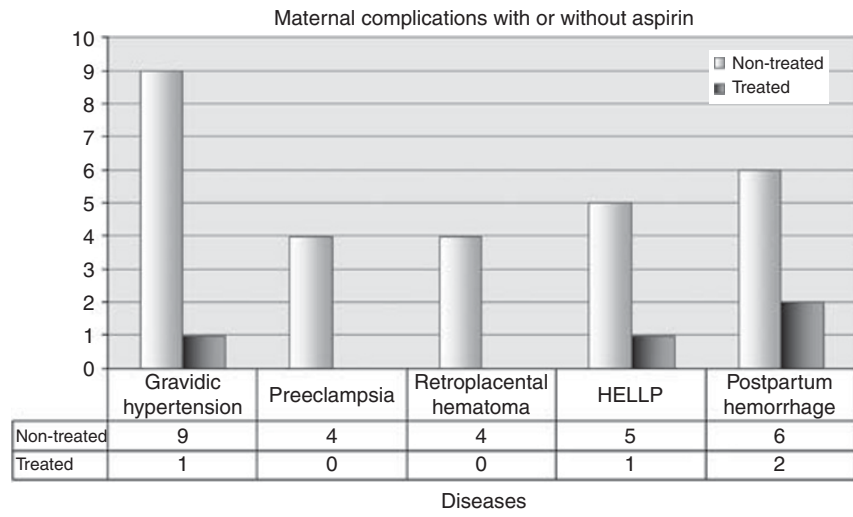

Figure 1 Aspirin treatment reduces the risk of maternal gravidic complications. Five types of gestational complications were followed. Gravidic hypertension and pre-eclampsia were considered to belong to the category of hypertensive diseases. Dark boxes correspond to treated cases and light gray boxes to non-treated cases. A significant improvement was visible in all situations (see text).

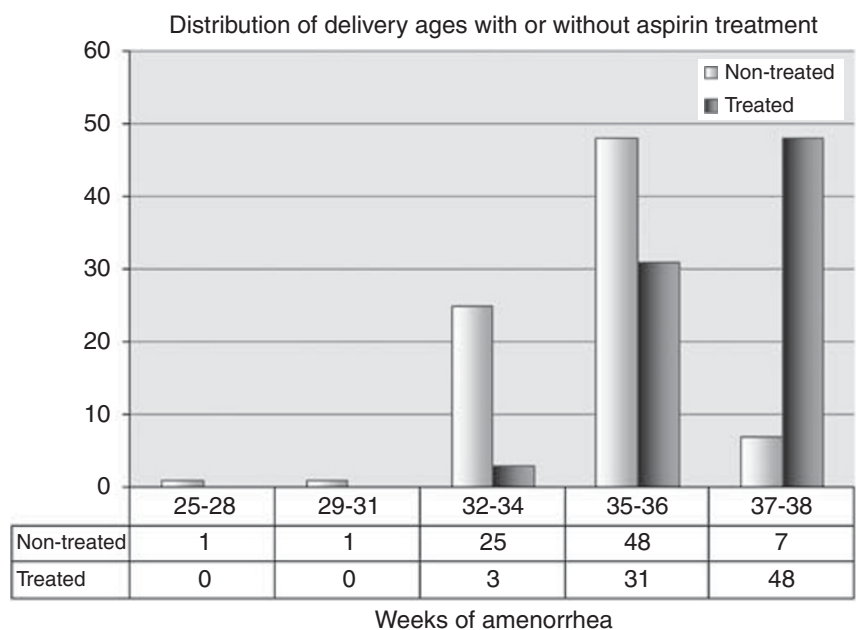

Figure 2 Aspirin treatment increases gestation length. On average, the treatment lengthened gestation by 12 days. The color code is the same as in Figure 1. Very premature babies were observed only in the non-treated group. In the treated group, $\sim 60 \%$ of the babies were born at 37-38 weeks of amenorrhea vs. 35-36 weeks in the non-treated group.

hemorrhage, respectively, were not significantly decreased in the treated group.

Under aspirin treatment, the average age for delivery was increased by 12 days

The distribution of ages at delivery is shown in Figure 2. The difference following treatment was highly significant after pooling of the three earliest age categories to reach a number of cases $>5$ in at least one group $\left(P<3.4 \times 10^{-11}\right)$. The average age at delivery was 36.6 weeks in the treated group vs. 34.7 weeks in the non-treated group. These results indicate that aspirin treatment delayed delivery by almost 2 weeks, with a complete absence of deliveries before 32 weeks in the treated group.

The weight of the newborn was increased under aspirin treatment Concomitantly with the increased age at delivery, the weight of the newborns was significantly increased $\left(P<6.8 \times 10^{-5}\right.$, Figure 3$)$. The

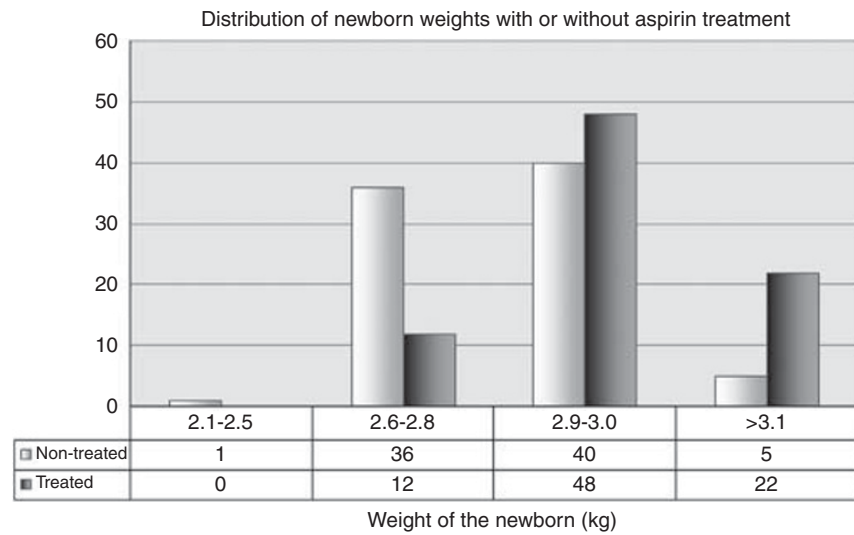

Figure 3 Aspirin treatment increases newborn weight. The color code is the same as in Figure 1. The average increase was $150 \mathrm{~g}$ (see text). This is essentially a consequence of the increase in gestation length.

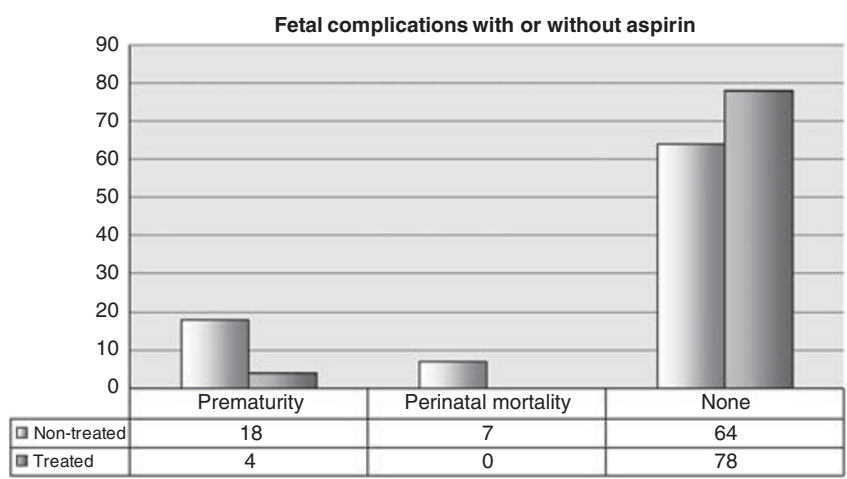

Figure 4 Aspirin treatment reduces deleterious fetal outcomes. The color code is the same as in Figure 1. Perinatal mortality was found at an elevated frequency in the non-treated group and among the premature infants. The specific labeling of the 10 cases of mortality with a hachured pattern is meant to take this into account, without counting them in the grand total.

average difference in baby weight was $\sim 150 \mathrm{~g}$. Analysis of intrauterine growth curves suggests that this difference may be directly attributable to the difference in gestation length. If the newborns are classified in two categories - up to $2.8 \mathrm{~kg}$ or above $2.9 \mathrm{~kg}$ - the improvement is clear, with an odds ratio of 4.8 (95\% confidence interval: 2.3-10.2).

\section{Fetal complications were reduced under aspirin treatment}

In the non-treated group, there were many more premature newborns than in the treated group. This difference correlated directly with gestation length. Quite notably, there was a strong and coincident reduction in perinatal mortality that was never observed in the non-treated group (Figure 4). Overall, the difference between treated and non-treated groups with respect to the number of fetal complications was highly significant $\left(P<1.3 \times 10^{-3}\right)$, with the risk of prematurity reduced $\sim 5$ fold (relative risk $(R R)=0.2295 \%$ confidence interval: $0.08-0.63$ ). No perinatal death was observed in the treated group; seven cases were observed in the non-treated group. The high incidence of perinatal deaths observed among the non-treated patients 
in this hospital condition $(\sim 8.5 \%)$ is nevertheless consistent with a more extensive study of more than 22000 births that yielded an estimate of $8.05 \%$ for perinatal death in nulliparous women in this region and these conditions.

\section{DISCUSSION}

Numerous studies have attempted to evaluate the beneficial effects of aspirin treatment in gestational hypertensive disorders, especially pre-eclampsia. Despite apparently conflicting results, it now appears that aspirin administered early has a genuinely positive effect on the outcome of such at-risk pregnancies. ${ }^{19}$ The study of Bujold and et al. ${ }^{18}$ confirms this hypothesis, because the papers dealing with early aspirin treatment consistently show a positive effect, with a relative risk ranging from 0.07 to 0.63 for pre-eclampsia and from 0.20 to 1 for IUGR. ${ }^{20-27}$ The present study is the largest to date with respect to the number of patients and the only one to administer aspirin at such an early point of gestation. In accordance with previous observations, we show that aspirin diminishes the risk of developing a hypertensive gravidic disease by threefold and delays the time of delivery by almost 2 weeks, thus increasing the birth weight by more than $150 \mathrm{~g}$. The risk of maternal complications was also reduced, and the risk of fetal complications was reduced by $\sim 10$-fold. The improvement in fetal outcomes is particularly striking in such a situation, where some weaknesses in the local health system lead to an important toll in terms of preterm mortality (see Figure 4).

The function of aspirin in preventing gestational hypertension diseases is not completely understood. Few studies have attempted to elucidate the role of aspirin by specifically designed experimentation. A recent study performed on cell culture with antioxidants, aspirin and heparin identified BeWo carcinoma cells as putative surrogates for trophoblasts owing to their PP13 increase following forskolin treatment; however, this study failed to detect specific effects of aspirin except for an increase in cell fusion induced by forskolin treatment. ${ }^{28}$ This may be due to the fact that the main targets of aspirin may not be cytotrophoblast- or choriocarcinoma-derived cell lines, but rather endothelial cells in the vascular part of the placenta. In the convoluted network of mechanisms involved in the onset of pre-eclampsia and other related disorders, ${ }^{29}$ thromboxane has been involved in placentation defects, which may lead to ischemia, placental thrombosis and platelet aggregation. It has been shown that low doses of aspirin selectively inhibit thromboxane A2 production by acting negatively on PTGS2 (COX2), the enzyme responsible for the conversion of arachidonic acid to thromboxane, and thus may prevent pregnancy-induced hypertension. ${ }^{22,30} \mathrm{~A}$ recent study on isolated trophoblasts showed that aspirin is able to decrease the release of thromboxane when the trophoblasts are isolated from normal but not from pre-eclamptic placentas. ${ }^{31}$ This again could suggest that when the pathology is established, the beneficial effects of aspirin are absent.

In conclusion, despite the need for large, double-blind, randomized studies, the present results support the idea that prescribing $100 \mathrm{mg}$ per day of aspirin to nulliparous women is a particularly efficient way of preventing pregnancy-associated hypertensive diseases. In the future, the development of genetic and/or serological tests may be helpful to decide which patients should be treated.

\section{CONFLICT OF INTEREST}

The authors declare no conflict of interest.
1 Goldenberg RL, Culhane JF, lams JD, Romero R. Epidemiology and causes of preterm birth. Lancet 2008; 371: 75-84.

2 Duley L. The global impact of pre-eclampsia and eclampsia. Semin Perinatol 2009; 33. 130-137.

3 Vikse BE, Irgens LM, Leivestad T, Skjaerven R, Iversen BM. Preeclampsia and the risk of end-stage renal disease. N Engl J Med 2008; 359: 800-809.

4 Magann EF, Washburne JF, Sullivan CA, Chauhan SP, Morrison JC, Martin Jr JN. Corticosteroid-induced arrest of HELLP syndrome progression in a marginally-viable pregnancy. Eur J Obstet Gynecol Reprod Biol 1995; 59: 217-219.

5 Armant DR, Kilburn BA, Petkova A, Edwin SS, Duniec-Dmuchowski ZM, Edwards HJ, Romero R, Leach RE. Human trophoblast survival at low oxygen concentrations requires metalloproteinase-mediated shedding of heparin-binding EGF-like growth factor. Development 2006; 133: 751-759.

6 Redman CW, Sargent IL. Placental stress and pre-eclampsia: a revised view. Placenta 2009; 30(Suppl A): S38-S42.

7 Blumenstein M, McMaster MT, Black MA, Wu S, Prakash R, Cooney J, McCowan LM, Cooper GJ, North RA. A proteomic approach identifies early pregnancy biomarkers for preeclampsia: novel linkages between a predisposition to preeclampsia and cardiovascular disease. Proteomics 2009; 9: 2929-2945

8 Auer J, Camoin L, Guillonneau F, Rigourd V, Chelbi ST, Leduc M, Laparre J, Mignot TM, Vaiman D. Serum profile in preeclampsia and intra-uterine growth restriction revealed by iTRAQ technology. J Proteomics 2010; 73: 1004-1017.

9 Chelbi ST, Mondon F, Jammes H, Buffat C, Mignot TM, Tost J, Busato F, Gut I, Rebourcet R, Laissue P, Tsatsaris V, Goffinet F, Rigourd V, Carbonne B, Ferre F, Vaiman D. Expressional and epigenetic alterations of placental serine protease inhibitors: SERPINA3 is a potential marker of preeclampsia. Hypertension 2007; 49: 76-83.

10 Maynard SE, Min JY, Merchan J, Lim KH, Li J, Mondal S, Libermann TA, Morgan JP, Sellke FW, Stillman IE, Epstein FH, Sukhatme VP, Karumanchi SA. Excess placental soluble fms-like tyrosine kinase 1 (sFlt1) may contribute to endothelial dysfunction, hypertension, and proteinuria in preeclampsia. J Clin Invest 2003; 111: 649-658.

11 Venkatesha S, Toporsian M, Lam C, Hanai J, Mammoto T, Kim YM, Bdolah $Y$, Lim KH, Yuan HT, Libermann TA, Stillman IE, Roberts D, D'Amore PA, Epstein FH, Sellke FW, Romero R, Sukhatme VP, Letarte M, Karumanchi SA. Soluble endoglin contributes to the pathogenesis of preeclampsia. Nat Med 2006; 12: 642-649.

12 Levine RJ, Lam C, Qian C, Yu KF, Maynard SE, Sachs BP, Sibai BM, Epstein FH, Romero R, Thadhani R, Karumanchi SA. Soluble endoglin and other circulating antiangiogenic factors in preeclampsia. N Engl J Med 2006; 355: 992-1005.

13 Thomas CP, Andrews JI, Liu KZ. Intronic polyadenylation signal sequences and alternate splicing generate human soluble FIt1 variants and regulate the abundance of soluble Flt1 in the placenta. FASEB J 2007; 21: 3885-3895.

14 Sela S, Itin A, Natanson-Yaron S, Greenfield C, Goldman-Wohl D, Yagel S, Keshet E. A novel human-specific soluble vascular endothelial growth factor receptor 1: celltype-specific splicing and implications to vascular endothelial growth factor homeostasis and preeclampsia. Circ Res 2008; 102: 1566-1574.

15 Thomas CP, Raikwar NS, Kelley EA, Liu KZ. Alternate processing of Flt1 transcripts is directed by conserved cis-elements within an intronic region of FLT1 that reciprocally regulates splicing and polyadenylation. Nucleic Acids Res 2010; 38: 5130-5140.

16 Darling M. Low-dose aspirin not for pre-eclampsia. Lancet 1998; 352: 342.

17 Haapsamo M, Martikainen H, Tinkanen H, Heinonen S, Nuojua-Huttunen S, Rasanen J. Low-dose aspirin therapy and hypertensive pregnancy complications in unselected IVF and ICSI patients: a randomized, placebo-controlled, double-blind study. Hum Reprod 2010; 25: 2972-2977.

18 Bujold E, Roberge S, Lacasse $Y$, Bureau M, Audibert F, Marcoux S, Forest JC, Giguere $Y$. Prevention of preeclampsia and intrauterine growth restriction with aspirin started in early pregnancy: a meta-analysis. Obstet Gynecol 2010; 116(2 Part 1): 402-414.

19 Carbillon L, Uzan S. Early treatment with low-dose aspirin is effective for the prevention of preeclampsia and related complications in high-risk patients selected by the analysis of their historic risk factors. Blood 2005; 105: 902; author reply 902-903.

20 August P, Helseth G, Edersheim T, Hutson J, Drusin M. Sustained release, low-dose aspirin ameliorates but does not prevent preeclampsia (PE) in a high risk population. Paper presented at the 9th International Congress of the International Society for the Study of Hypertension, Sydney, Australia, 1994.

21 Beaufils M, Uzan S, Donsimoni R, Colau JC. Prevention of pre-eclampsia by early antiplatelet therapy. Lancet 1985; 1: 840-842.

22 Benigni A, Gregorini G, Frusca T, Chiabrando C, Ballerini S, Valcamonico A, Orisio S, Piccinelli A, Pinciroli V, Fanelli R, Gastaldi A, Remuzzi G. Effect of low-dose aspirin on fetal and maternal generation of thromboxane by platelets in women at risk for pregnancy-induced hypertension. N Engl J Med 1989; 321: 357-362.

23 Dasari R, Narang A, Vasishta K, Garewal G. Effect of maternal low dose aspirin on neonatal platelet function. Indian Pediatr 1998; 35: 507-511.

24 Ebrashy A, Ibrahim M, Marzook A, Yousef D. Usefulness of aspirin therapy in high-risk pregnant women with abnormal uterine artery Doppler ultrasound at 14-16 weeks pregnancy: randomized controlled clinical trial. Croat Med J 2005; 46: 826-831.

25 Hermida RC, Ayala DE, Iglesias M, Mojon A, Silva I, Ucieda R, Fernandez JR. Timedependent effects of low-dose aspirin administration on blood pressure in pregnant women. Hypertension 1997; 30(3 Part 2): 589-595.

26 Tulppala M, Marttunen M, Soderstrom-Anttila V, Foudila T, Ailus K, Palosuo T, Ylikorkala O. Low-dose aspirin in prevention of miscarriage in women with unexplained 
or autoimmune related recurrent miscarriage: effect on prostacyclin and thromboxane A2 production. Hum Reprod 1997; 12: 1567-1572.

27 Vainio M, Kujansuu E, Iso-Mustajarvi M, Maenpaa J. Low dose acetylsalicylic acid in prevention of pregnancy-induced hypertension and intrauterine growth retardation in women with bilateral uterine artery notches. BJOG 2002; 109: 161-167.

28 Orendi K, Gauster M, Moser G, Meiri H, Huppertz B. Effects of vitamins C and E, acetylsalicylic acid and heparin on fusion, beta-hCG and PP13 expression in BeWo cells. Placenta 2010; 31: 431-438.

29 Rigourd V, Chelbi S, Chauvet C, Rebourcet R, Barbaux S, Bessieres B, Mondon F, Mignot TM, Danan JL, Vaiman D. Re-evaluation of the role of STOX1 transcription factor in placental development and preeclampsia. J Reprod Immunol 2009; 82: 174-181.

30 Schiff E, Peleg E, Goldenberg M, Rosenthal T, Ruppin E, Tamarkin M, Barkai G, Ben-Baruch G, Yahal I, Blankstein J, Goldman B, Maschiach S. The use of aspirin to prevent pregnancy-induced hypertension and lower the ratio of thromboxane A2 to prostacyclin in relatively high risk pregnancies. $N$ Engl J Med 1989; 321: 351-356.

31 Zhao S, Gu Y, Lewis DF, Wang Y. Predominant basal directional release of thromboxane, but not prostacyclin, by placental trophoblasts from normal and preeclamptic pregnancies. Placenta 2008; 29: 81-88. 